\title{
Minimize Reactive Power Losses of Dual Active Bridge Converters using Unified Dual Phase Shift Control
}

\author{
Huiqing Wen ${ }^{\dagger}$ and Bin $\mathrm{Su}^{*}$
}

\begin{abstract}
This paper proposed an unified dual-phase-shift (UDPS) control for dual active bridge (DAB) converters in order to improve efficiency for a wide output power range. Different operating modes of UDPS are characterized with respect to the reactive current distribution. The proposed UDPS has the same output power capability with conventional phase-shift (CPS) method. Furthermore, its implementation is simple since only the change of the leading phase-shift direction is required for different operating power range. The proposed UDPS control can minimize both the inductor rms current and the circulating reactive current for various voltage conversion ratios and load conditions. The optimal phase-shift pairs for two bridges of DAB converter are derived with respect to the comprehensive reactive power loss model, including the reactive components delivered from the load and back to the source. Simulation and experimental results are illustrated and explained with details. The effectiveness of the proposed method is verified in terms of reactive power losses minimization and efficiency improvement.
\end{abstract}

Keywords: DC-DC converter, Phase shift control, Reactive power loss, Power characterization

\section{Nomenclature}

d Voltage conversion ratio

$N \quad$ Transformer turn ratio

$i_{\text {in }} \quad$ Input current

$i_{o} \quad$ Output current

$\beta_{i} \quad$ Zero-crossing instant of the inductor current $i_{L}$

$\delta_{i} \quad$ Phase shift angle

$V_{D S 1} \quad$ Voltage drop of the MOSFETs in bridge 1

$V_{D S 2} \quad$ Voltage drop of the MOSFETs in bridge 2

$d_{\text {DPS_III }}$ Boundary of DPS_I and DPS_II

$d_{\text {DPS_IIIIII }}$ Boundary of DPS_II and DPS_III

\section{Introduction}

The dual active bridge (DAB) converter has been widely used in HVDC and MVDC grids [1], DC distributed power system [2], solid-state transformer [3, 4], automotive [5], and aerospace applications [6] since it shows advantages of bidirectional power transfer, compact design, easy implementation, and inherent soft switching feature [7-9]. Usually the modulation strategy adopted is the conventional phase shift (CPS) control, where the phase shift determines directly both the power flow direction and the amplitude of the delivery power. The schematic of DAB

$\dagger \quad$ Corresponding Author: Dept. of Electrical and Electronic Eng., Xi'an Jiaotong-Liverpool University, China. / State Key Laboratory of Electrical Insulation and Power Equipment, Xi'an Jiaotong University, China. (Huiqing.Wen@xjtlu.edu.cn)

* Hangzhou Electric Power Bureau, State Grid Corporation of China Hangzhou, China. (Supeninsula@gmail.com)

Received: December 10, 2015; Accepted: October 11, 2016 converter with CPS control and key waveforms is shown in Fig. 1. As shown the blue shaded areas in Fig. 1(b), the input current $i_{\text {in }}$ is not always in phase with the primary voltage $V_{T I}$. Similarly, the output current $i_{o}$ is not always in phase with the secondary voltage $V_{T 2}$, as shown the orange shaded areas in Fig. 1(b). Especially when the voltage conversion ratio is not unity, CPS might result in high

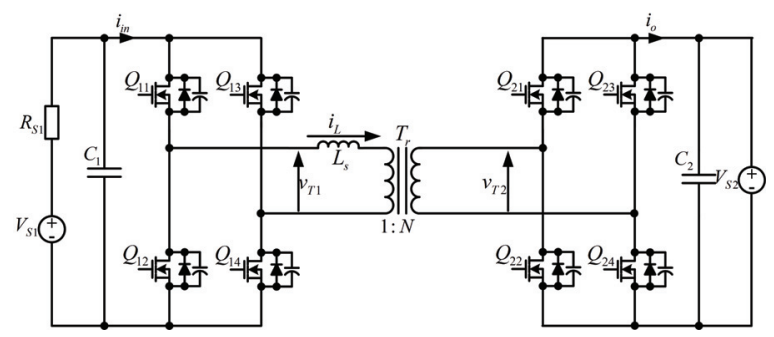

(a)

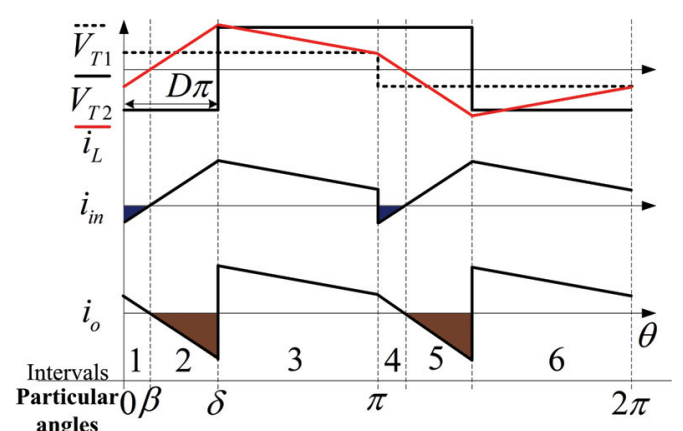

(b)

Fig. 1 Circuit schematic of DAB converter topology and typical waveforms with CPS: (a) Circuit Schematic; (b) Typical waveforms 
circulating current that flows through the power devices and magnetic components. Furthermore, by using the CPS control, the soft-switching feature might also be lost under some scenarios $[7,10,11]$.

To address this issue, several advanced strategies are discussed previously, including the combination of phaseshift and pulse width modulation (PSPWM) control [12], dual phase-shift (DPS) control [13], and its extended version [14]. However, a comprehensive investigation the power flow characterization of each operating mode by using these strategies has rarely been reported and the criteria in determining the control variables such as duty cycles or phase shift values has not been properly addressed. Besides, various high frequency link resonant tanks, including the LC resonant tank [15], the LLC-based resonant tank [16], and CLLC-based resonant tank, have been added in order to extend the range of soft switching [17]. However, there are mainly two concerns in adopting the resonant tanks: one is the resonant electrical stresses and the other is the increased cost due to additional components such as capacitors or inductors. In fact, reactive current or reactive power contributes significantly the conduction loss of power devices and connectors. Besides, the magnetic losses of inductor and transformer are also increased. In prior researches, "reactive power" is just defined by the portion of reactive power delivered back to the source [8]. However, the definition of "reactive power" is incomplete since the reactive power component delivered from the load is not considered. The similar question in reactive current analysis was founded in [18]. Since the analysis of reactive power is incomplete, the optimization by using reactive power is inconsistent with the results using other optimization objectives such as the minimum inductor peak or rms current [19]. In [2], the reactive power losses of DAB converter by using various strategies are analyzed and adopted as the optimization objective. However, this modulation has a limited maximum output power, which is half of that with CPS. Thus, an unified dual phase shift (UDPS) control is necessary to cover a whole range of output power. Another problem is that the advanced phase-shift methods have at least two variables and several operating modes, which show totally different power characterizations, including the output power range and the reactive current distribution, the power characterization analysis of each operating mode is normally ignored in prior research $[2$, $13,14,20]$.

To address these problems, in this paper, the UDPS control scheme is proposed and its operating modes are analyzed with their power characteristics. Furthermore, the power loss distribution is illustrated with their mathematical expressions. Simulation model was built and a scale-down experimental prototype was established. The effectiveness of the proposed method is verified by both simulation and experimental results in terms of reactive power losses minimization and efficiency improvement.
This paper is organized as follows. In Section II, the dual phase shift control is introduced with different operating modes analysis. In order to achieve a complete output power range, an unified dual phase shift control is proposed and discussed in Section III. Then the experimental results are illustrated and explained in Section IV. Finally Conclusions are drawn in Section V.

\section{Reactive Power Loss}

In this section, the reactive power loss is defined as the conduction loss of the semiconductor power switches considering the inherent relationship between the reactive power loss and the reactive current. Since different operating modes present totally different reactive current distribution, here the reactive power loss model of DAB converter is discussed by considering each operating mode of dualphase-shift (DPS) strategy.

\subsection{Power characterization analysis}

The DPS scheme includes two phase-shift variables symbolized as $D_{1}$ and $D_{2}$. Fig. 2 shows typical operating waveforms of DAB converters modulated by the DPS. $D_{l}$ represents the ratio for the leading phase shift, which is opposite to the power flow direction (from $V_{S 1}$ to $V_{S 2}$ ). $D_{2}$ is the lag phase-shift ratio.

Considering that the interval between $\delta_{1}$ and $\delta_{2}$

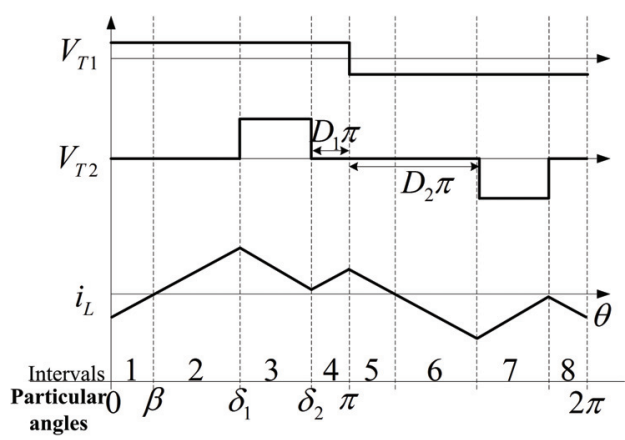

(a)

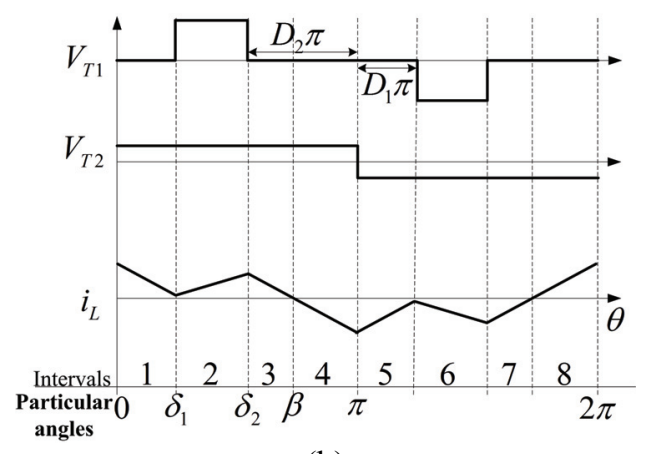

(b)

Fig. 2. Typical waveforms with DPS control: (a) boost operation; (b) buck operation [12] 
represents the period of positive output voltage (boost operation), the expression for the average output power is

$$
P_{o}=\frac{V_{T_{2}}}{N} \int_{\delta_{1}}^{\delta_{2}} i_{L} d t=\frac{V_{T_{2}}\left(\delta_{2}-\delta_{1}\right)}{2 N}\left[i_{L}\left(\delta_{1}\right)+i_{L}\left(\delta_{2}\right)\right] \geq 0
$$

Thus,

$$
\begin{gathered}
i_{L}\left(\delta_{1}\right)+i_{L}\left(\delta_{2}\right)=2\left(D_{2}-D_{1}\right) \geq 0 \\
D_{2} \geq D_{1}
\end{gathered}
$$

Taking the deviation of the output power $P_{o}$ with $D_{l}$ and $\mathrm{D}_{2}$ respectively, we obtain

$$
\begin{aligned}
& \left(d P_{o}\right) /\left(d D_{1}\right)=2 D_{1}-1 \quad \forall 0 \leq D_{1} \leq D_{2} \leq 1 \\
& \left(d P_{o}\right) /\left(d D_{2}\right)=1-2 D_{2} \quad \forall 0 \leq D_{1} \leq D_{2} \leq 1
\end{aligned}
$$

Combining (4), (5) and the constraint of $0 \leq D_{1}+D_{2} \leq 1$, the maxima of the output power is derived as

$$
P_{\text {max }}=0.25 \forall D_{1}=0 \wedge D_{2}=0.5
$$

Fig. 3 illustrates three modes with DPS control and their main difference is the distribution of reactive current. The blue shaded areas represent the reactive current components delivered back to the source. The orange shaded areas represent the reactive current that is delivered from the load.

\subsection{Reactive power loss analysis}

Fig. 3(b) shows that the first inductor-current zero crossing angle by using DPS_II $\beta_{D P S_{-} I I_{1} 1}$ is within the range of $\left[0, \delta_{1}\right]$, thus, the slope of $i_{L}$ during this interval can be expressed as

$$
k_{1}=V_{S_{1}} / L_{s}
$$

The first inductor-current zero crossing angle meets the relationship:

$$
i_{L(0)}+k_{1} \beta_{D P S_{-} I I-1} / 2 \pi f=0
$$

The inductor current analytical expressions at the defined switching angles can be derived. Then, the following equation can be obtained:

$$
\frac{V_{S_{1}}\left(-d D_{1}-d D_{2}+d-1\right)}{4 L_{s} f_{s}}+\frac{V_{S_{1}}}{L_{s}} \cdot \frac{\beta_{D P S_{-} I I_{-} 1}}{2 \pi f}=0
$$

Thus, the first inductor-current zero crossing angle $\beta_{D P S_{-} I I_{-} 1}$ can be expressed as follows:

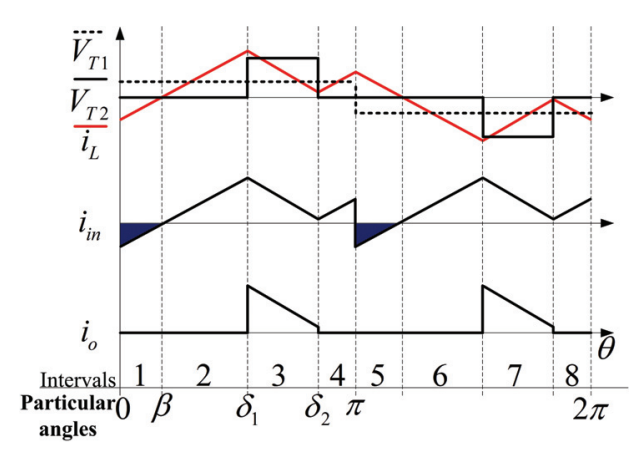

(a)

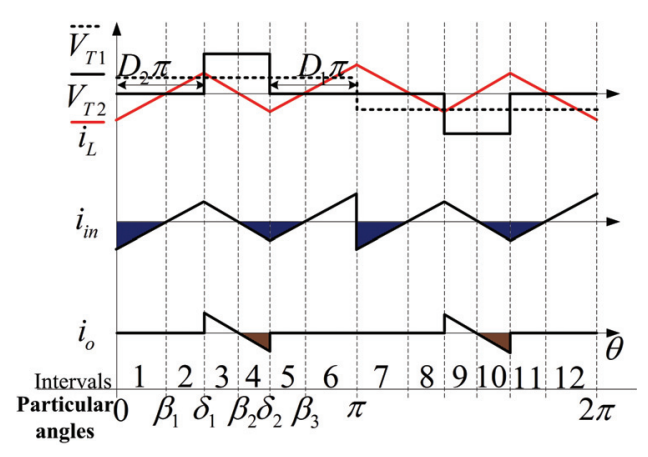

(b)

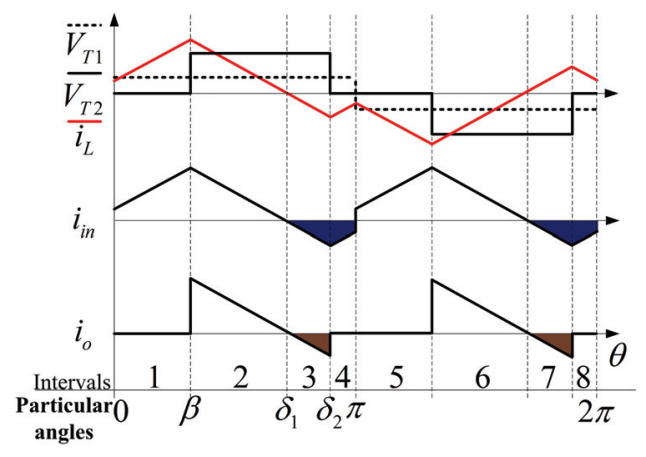

(c)

Fig. 3 Modes analysis with the DPS for boost operation:

(a) DPS_I; (b) DPS _II; (c) DPS _III [2]

$$
\beta_{D P S_{-} I I_{-} 1}=\left(d D_{1}+d D_{2}-d+1\right) \pi / 2
$$

The second inductor-current zero crossing angle $\beta_{D P S_{-} I I_{-} 2}$ should be within the range of $\left[\delta_{1}, \delta_{2}\right]$. The slope of $i_{L}$ during this interval can be expressed as

$$
k_{2}=(1-d) V_{S_{1}} / L_{s}
$$

The second inductor-current zero crossing angle $\beta_{D P S_{-} I I_{-} 2}$ meets the relationship:

$$
i_{L\left(\delta_{1}\right)}+k_{2} \beta_{D P S_{-} I I_{-} 2} / 2 \pi f=0
$$

Then, after deriving the analytical expressions for the inductor current at different switching angles, the following 
equation can be obtained:

$$
\begin{gathered}
\frac{V_{S_{1}}\left(-d D_{1}-d D_{2}+d-1+2 D_{2}\right)}{4 L_{s} f_{s}}+\frac{(1-d) V_{S_{1}}}{L_{s}} . \\
\frac{\left(\beta_{D P S_{-} I I_{-} 2}-D_{2} \pi\right)}{2 \pi f}=0
\end{gathered}
$$

Thus, the second inductor-current zero crossing angle $\beta_{D P S_{-} I I_{-} 2}$ can be expressed as:

$$
\beta_{D P S_{-} I I_{-} 2}=\left(d D_{2}-d D_{1}+d-1\right) \pi /[2(d-1)]
$$

Similarly, the third inductor-current zero crossing angle $\beta_{D P S_{-} I I_{-} 3}$ can also be obtained as

$$
\beta_{D P S_{-} I I_{-} 3}=\left(-d D_{1}-d D_{2}+d+1\right) \pi / 2
$$

Considering the dynamics of $i_{L}$ shown in Fig. 3, the inductor-current zero crossing angles using DPS_I and DPS_III are expressed as

$$
\begin{gathered}
\beta_{D P S_{-} I}=\left(d D_{1}+d D_{2}-d+1\right) \pi / 2 \\
\beta_{D P S_{-} I I I}=\left(d D_{2}-d D_{1}+d-1\right) \pi /[2(d-1)]
\end{gathered}
$$

The expressions for the reactive current duty-ratio $D_{B 1}$ and $D_{B 2}$ with DPS for boost operation are shown in Table 1. Table 2 shows the corresponding expressions for the reactive power loss $P_{R L B B 1}$ and $P_{R L_{-} B 2}$. Where, $V_{D S 1}$ and $V_{D S 2}$ symbol the voltage drop of the power devices for two bridges of DAB converters.

For boost operation, the boundary of DPS_I and DPS_II

Table 1. Expressions of reactive current time duty ratio with DPS for boost operation (p.u.)

\begin{tabular}{c|c|c}
\hline Modes & $D_{B 1}$ & $D_{B 2}$ \\
\hline DPS_I & $\left(d D_{1}+d D_{2}-d+1\right) / 2$ & 0 \\
\hline DPS_II & $\frac{\left(d D_{1}-d D_{2}+d-1\right)}{2(d-1)}$ & $\frac{\left(2 D_{1}-d D_{1}-d D_{2}+d-1\right)}{2(d-1)}$ \\
\hline DPS_III & $\frac{\left(d D_{1}-d D_{2}+d-1\right)}{2(d-1)}$ & $\frac{\left(2 D_{1}-d D_{1}-d D_{2}+d-1\right)}{2(d-1)}$ \\
\hline
\end{tabular}

can be determined considering that it is dependent on the inductor current at switching angle $\delta_{2}$.

$$
i_{L\left(\delta_{2}\right)}=-d+d D_{2}+d D_{1}-2 D_{1}+1=0
$$

Thus,

$$
d_{\text {DPS_I_II_boost }}=\left(1-2 D_{1}\right) /\left(1-D_{1}-D_{2}\right)
$$

The boundary of DPS_II and DPS_III can also be determined with the expression below

$$
d_{D P S_{-} I I_{-} I I I_{-} \text {boost }}=1 /\left(1-D_{1}-D_{2}\right)
$$

For buck operation, similarly, the expressions for the boundary of different modes with DPS are

$$
\begin{gathered}
d_{D P S_{-} I_{-} I I_{-} \text {buck }}=\left(1-D_{1}-D_{2}\right) /\left(1-2 D_{1}\right) \\
d_{D P S_{-} I I_{-} I I I_{-} \text {buck }}=1-D_{1}-D_{2}
\end{gathered}
$$

Fig. 4(a) shows the comparison of phase shift distribution ratio using $P_{R L_{-} \min }$ and $I_{r m s_{\text {min }}}$ respectively. Fig. 4(b) illustrate the derived inductor rms current difference ratio by using two methods. The inductor rms current difference ratio $I_{r m s \_ \text {diff }} \%$ by using CPS and DPS method is defined by

$$
I_{r m s_{-} \text {diff }} \%=\frac{I_{r m s_{-} \mathrm{D} P S}-I_{r m s_{-} \mathrm{C} P S}}{I_{r m s_{-} \mathrm{C} P S}} \times 100 \%
$$

Although the phase-shift pairs obtained by the method of $P_{R L_{-} \min }$ is not completely consistent with that obtained by $I_{r m s \text { min }}$, as indicated in Fig. 4(b). However, the derived rms current difference ratio for the whole power range is within $2.5 \%$. Fig. 4(c) illustrates the derived reactive power difference ratio obtained by $P_{R L \text { min }}$ and $Q_{L \text { min }}$ under " $d=1.5 "$. The derived reactive power difference ratio for the whole operating range is within $6 \%$. Thus, in this paper, the reactive power loss is defined and adopted as the optimization objective. The optimal phase shift pairs are determined with this objective. Fig. 4(d) illustrates the comparison of $P_{R L} / P_{o}$ using two modulation strategies of CPS and DPS control with respect to $P_{o}$ under " $d=1.5$ ". The main parameters are $V_{D S 1}=1 \mathrm{~V}, V_{D S 2}=3 \mathrm{~V}$, input

Table 2. Reactive power losses expressions with DPS for boost operation (p.u.)

\begin{tabular}{c|c|c}
\hline Modes & $P_{R L B 1}$ & $P_{R L B 2}$ \\
\hline DPS_I & {$\left[V_{D S 1}\left(d D_{1}+d D_{2}-d+1\right)^{2}\right] /\left(2 V_{S 1}\right)$} & 0 \\
\hline DPS_II & $V_{D S 1}\left[\begin{array}{l}\left.(d-1)\left(d D_{1}+d D_{2}-d+1\right)^{2}+\right] \\
d\left(d D_{1}-d-2 D_{1}+d D_{2}+1\right)^{2}\end{array}\right] /\left[2 V_{1}(d-1)\right]$ & $V_{D S 2}\left(\begin{array}{l}d D_{1}+d D_{2} \\
-2 D_{1}-d+1\end{array}\right)^{2} /\left[2 N V_{1}(d-1)\right]$ \\
\hline DPS_III & $V_{D S 1}\left(\begin{array}{l}-3 d^{2} D_{1}^{2}+4 d D_{1}^{2}-2 d^{2} D_{1} D_{2} \\
+2 d^{2} D_{1}-2 d D_{1}+d^{2} D_{2}^{2}-2 d^{2} D_{2} \\
+2 d D_{2}+d^{2}-2 d+1\end{array}\right) /\left[2 V_{1}(d-1)\right]$ & $V_{D S 2}\left(\begin{array}{l}d D_{1}+d D_{2} \\
-2 D_{1}-d+1\end{array}\right)^{2} /\left[2 N V_{1}(d-1)\right]$ \\
\hline
\end{tabular}


voltage $V_{S I}=20 \mathrm{~V}$, and transformer turns ratio $N=6$. It shows that the $P_{R L} / P_{o}$ with DPS is reduced far below the pre-define $2 \%$ reference for a majority range of the output power. The green line in Fig. 4(d) represents the $2 \%$ reference. Fig. 4(d) shows that the range of $P_{R L} / P_{o}$ below the reference line can be significantly enlarged with operating modes of DPS. Furthermore, among three operating modes, DPS_II is best option in terms of the low $P_{R L} / P_{o}$ value and the wide operating range.

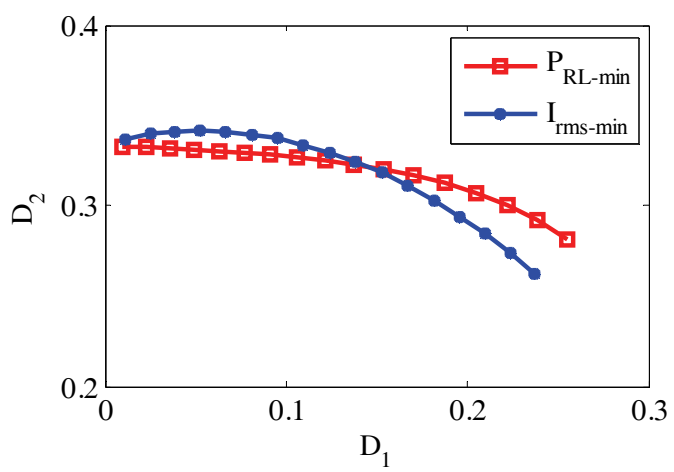

(a)

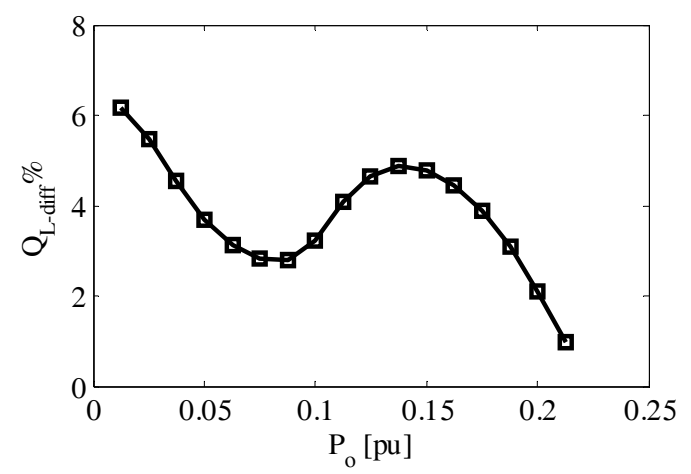

(c)

\section{Unified Phase Shift Control}

Considering that the DPS scheme has a limited maximum output power, which is half of that with CPS according to the expression (6), the range of leading phaseshift variable $D_{1}$ with DPS needs to be extended to achieve the a wider output power range [2]. Thus, an unified DPS scheme for DAB converter is developed, specifically, for high-power ranges. Similar to the DPS, the proposed

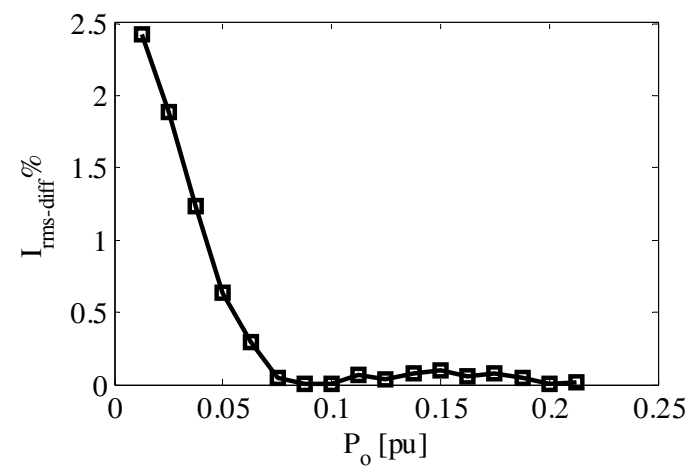

(b)

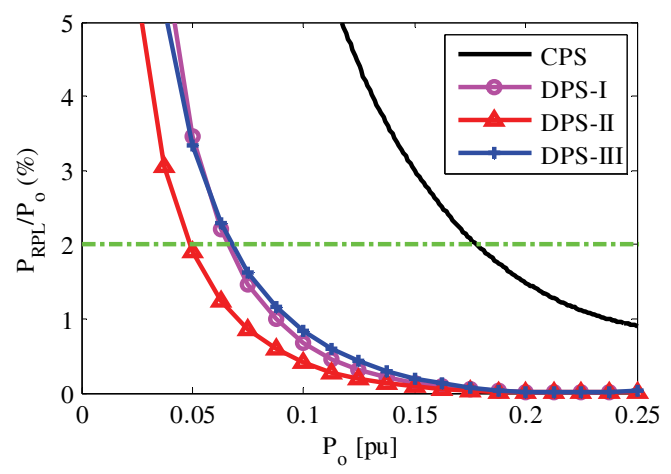

(d)

Fig. 4. Phase-shift pairs distribution with DPS and performance evaluation: (a) distribution of phase shift pairs; (b) difference ratio of the inductor rms current using $P_{R_{L} \text { min }}$ and $I_{r m s \text { min }}$; (c) reactive power difference ratio using $P_{R L_{-} \text {min }}$ and $Q_{L \text { min }}$; (d) $P_{R L} / P_{o}$ with respect to $P_{o}$ under " $d=1.5$ "
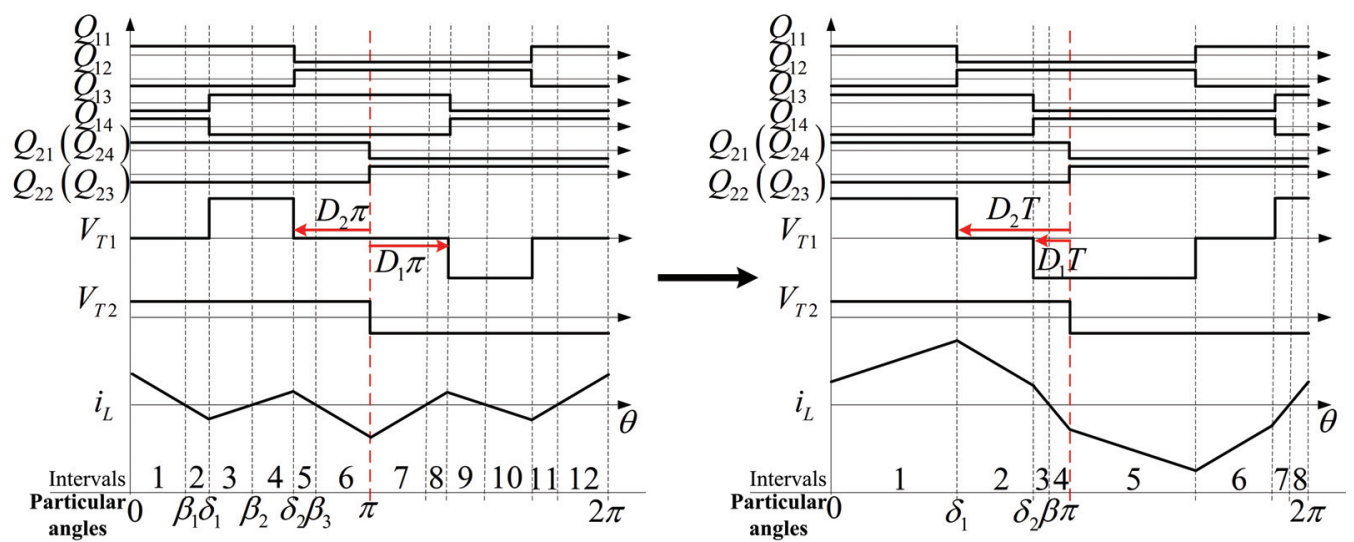

Fig. 5. UDPS scheme to achieve the full power delivery capability 
unified DPS has two phase-shift variables, namely $D_{l}$, and $D_{2}$. Totally there are four different modes according to different combinations of $\left(D_{1}, D_{2}\right)$. Compared with the CPS, the practical implementation becomes difficult considering more phase-shift variables and operating modes. Thus, here a simplified UDPS control scheme is proposed for different power regions in practical implementation and the detailed operating waveforms are illustrated in Fig. 5.

In low-power range, the direction of leading phase-shift $D_{1}$ is arranged to be opposite to the direction of phase-shift $D_{2}$ and the mode of DPS II with multiple zero crossing points of inductor current is adopted considering its capability of low reactive power loss.

In high-power range, the direction of leading phase-shift $D_{1}$ is set to the same as the direction of phase-shift $D_{2}$. This variation of DPS is named as VDPS. The expressions for the inductor current at different switching angles and the corresponding output power are shown in Table 3 for both buck and boost operations.

For DAB converters with two DC voltage sources, the

Table 3. Expressions for the current at different switching angles and output power with VDPS (pu)

\begin{tabular}{c|c|c}
\hline $\begin{array}{c}\text { Switching } \\
\text { Angles }\end{array}$ & $d \geq 1$ & $d<1$ \\
\hline$i_{L}(0)$ & $-d D_{1}-d D_{2}+d-1$ & $D_{1}+D_{2}+d-1$ \\
\hline$i_{L}\left(\delta_{1}\right)$ & $d D_{1}-d D_{2}+d-1+2 D_{1}$ & $D_{1}-D_{2}-d+1+2 d D_{2}$ \\
\hline$i_{L}\left(\delta_{2}\right)$ & $d-d D_{2}+d D_{1}+2 D_{2}-1$ & $D_{1}-D_{2}-d+2 d D_{1}+1$ \\
\hline$i_{L}(\pi)$ & $d D_{1}+d D_{2}-d+1$ & $-D_{1}-D_{2}-d+1$ \\
\hline$P_{o}$ & $D_{1}+D_{2}-D_{1}^{2}-D_{2}^{2}$ & $D_{1}+D_{2}-D_{1}^{2}-D_{2}^{2}$ \\
\hline
\end{tabular}

boost operation in one direction corresponds to the buck operation in the other direction. Thus, the proposed UDPS scheme can be applied for bi-directional power flow. The complete operating modes for buck operation regarding the reactive current distribution are illustrated in Fig. 6. Table 4 shows the expressions of the reactive power losses with buck operation. According to the proposed method of achieving minimum reactive power loss $P_{R L \text { min }}$, VDPS_I is determined as the optimal operating mode.

The phase-shift pairs of the UDPS scheme are determined by the equations as

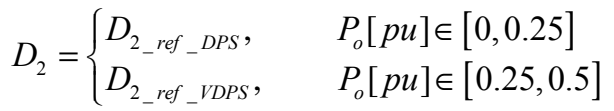

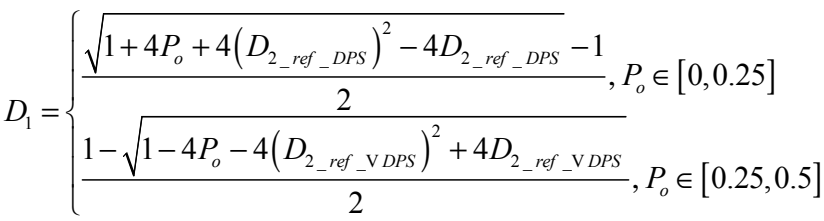

Fig. 7 illustrates optimal phase shift pairs distribution of UDPS versus power for " $d=0.5$ ". The black line indicates the boundary of DPS and VDPS. Across this boundary, the direction of leading phase-shift $D_{l}$ is changed from negative to positive. Fig. 7 also shows that the optimal $D_{2}$ by $P_{R L_{-} \min }$ locates mainly in the range of $(0.4,0.5)$.

Fig. 8 illustrates simulated waveforms of " $v_{T 1}$ ", " $i_{L}$ ", " $i_{i n}$ ", and " $i_{o}$ " under " $d=0.5$ " and " $P_{o}[$ p.u. $]=0.15$ ". As shown in Fig. 8(a), the primary voltage $v_{T l}$ is of three-level voltage due to two phase-shift operations, namely $D 1$ and $D 2$. As

Table 4. Reactive power losses with VDPS for buck operation (p.u.)

\begin{tabular}{c|c|c}
\hline Modes & PRL_B1 & PRL_B2 \\
\hline VDPS_I & $\frac{V_{C E 1}\left(D_{1}-D_{2}-d+2 d D_{1}+1\right)^{2}}{4 V_{S 1}(d+1)}$ & $\frac{V_{C E 2}\left(D_{1}+D_{2}+d-1\right)^{2}}{4 N V_{S 1}(d+1)}$ \\
\hline VDPS_II & $\frac{V_{C E 1}\left(D_{1}+D_{2}+d-1\right)^{2}}{4 V_{S 1}(1-d)}+\frac{V_{C E 1}\left(d D_{1}-D_{2}-d+1\right) D_{1}}{V_{S 1}}$ & $\frac{V_{C E 2}\left(D_{1}+D_{2}+d-1\right)^{2}}{4 N V_{S 1}(1-d)}$ \\
\hline
\end{tabular}

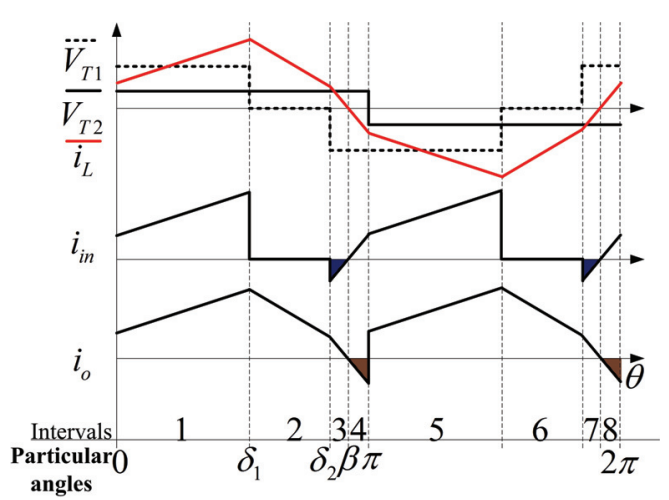

(a)

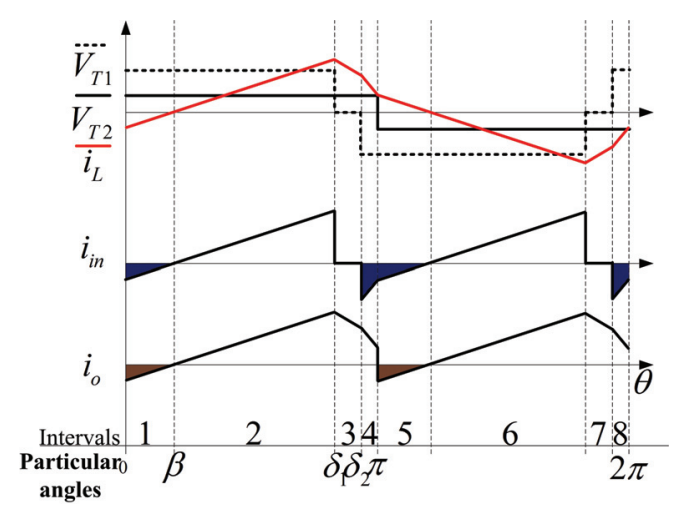

(b)

Fig. 6. Modes analysis of DAB converters with VDPS for buck operation: (a) VDPS_I; (b) VDPS III. 
shown in Fig. 8(b), the peak and rms values of $i_{L}$ are decreased with UDPS compared with that with CPS. The comparison of input current is shown in Fig. 8(c) and it indicates that the reactive current component delivered back to the source is minimized close to zero. Fig. 8(d) indicates that the reactive current delivered from the load is significantly reduced with UDPS control.

Fig. 9 shows the performance comparison with the UDPS and CPS scheme in terms of the rms current and the $P_{R L} / P_{o}$ under " $d=0.5$ " for a whole operating range of power. The parameters used in the analysis are shown as: $V_{D S 1}=0.2 \mathrm{~V}, V_{D S 2}=3 \mathrm{~V}, V_{S I}=20 \mathrm{~V}, N=6$. The values of $P_{R L} / P_{o}$

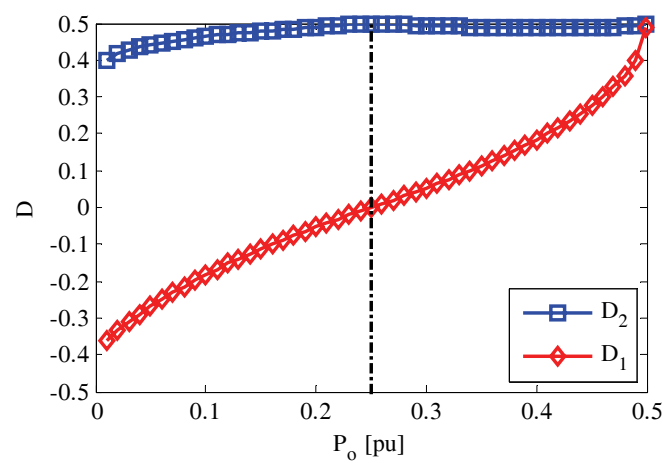

Fig. 7. Optimal phase-shift pairs distribution with UDPS versus the whole output power range under " $\mathrm{d}=0.5$ "

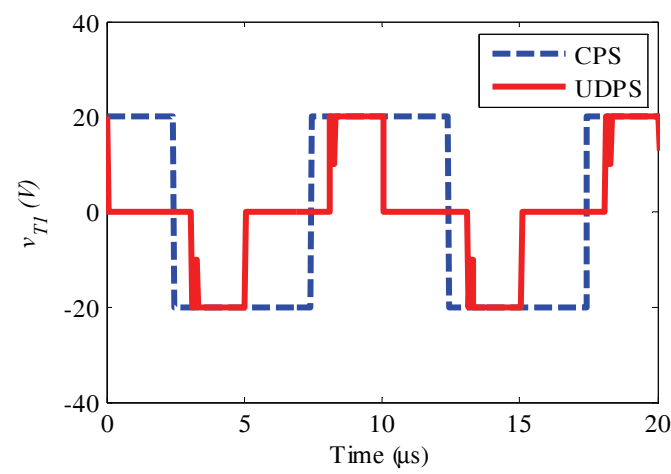

(a)

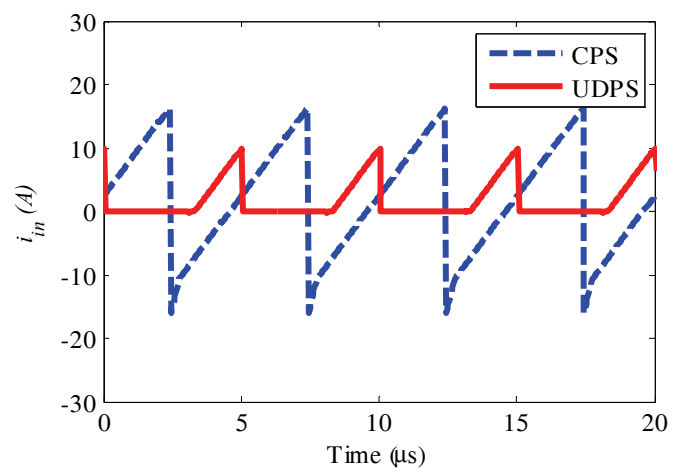

(c) using the UDPS scheme are significantly reduced and the effective output power range corresponding to the $P_{R L} / P_{o}$ less than $2 \%$ is extended from $(0.25,0.5)$ to $(0.02,0.5)$, as illustrated in Fig. 9(a). The rms current is significantly reduced, which contributes the minimization of the power losses. The inductor rms current values by using UDPS and CPS are compared and shown in Fig. 9(b). It indicated that the rms value of $i_{L}$ has been significantly reduced by using UDPS for the whole operating power range. Thus, the power device conduction losses and the magnetic losses will be reduced correspondingly.

\section{Power Loss Analysis}

The total power loss is divided into several parts: conduction loss of the power devices $P_{\text {cond }}$, switching loss of devices $P_{s w}$, the loss of transformer and inductor $P_{m a g}$ and others. For the conduction loss $P_{\text {cond }}$, the calculation expression is:

$$
P_{\text {cond }}=\left(2 R_{Q 11}+2 R_{Q 21} / N^{2}\right) I_{L}^{2}
$$

where $R_{Q 11}$ and $R_{Q 21}$ represent the conduction resistance of the primary and secondary MOSFETs, respectively.

For the switching loss $P_{s w}$, firstly the soft-switching

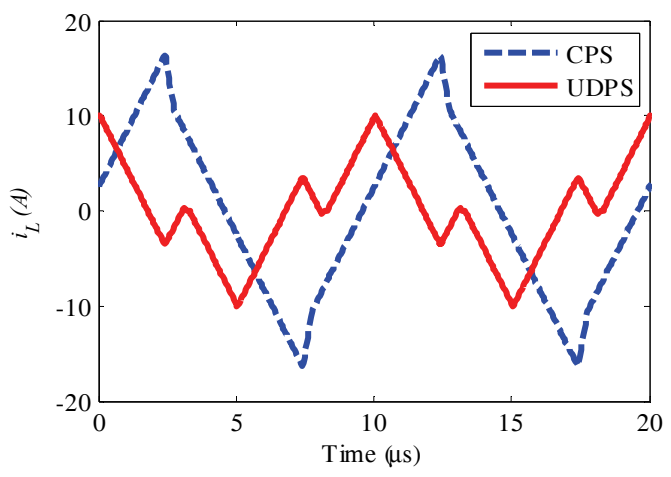

(a)

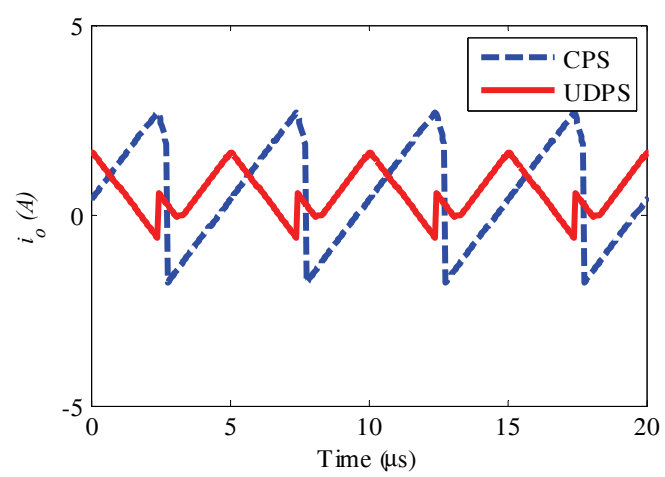

(d)

Fig. 8. Performance comparison of two modulation strategies of CPS and UDPS under " $d=0.5$ " and Po[p.u.] $=0.15$ : (a) vT1; (b) iL; (c) iin; (d) io. 


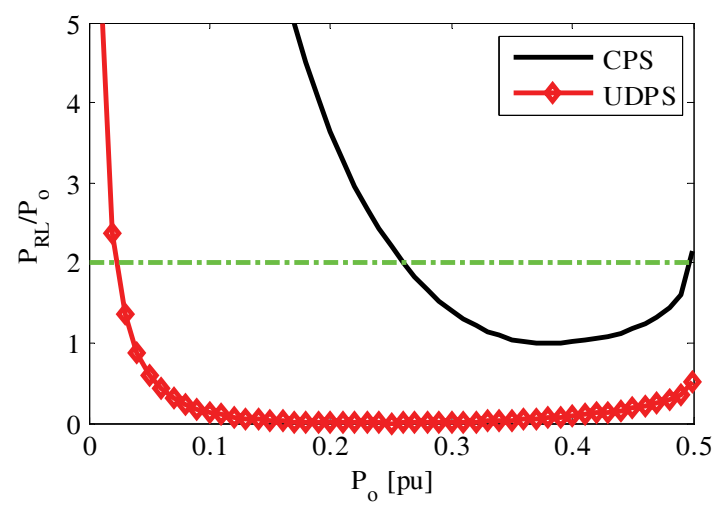

(a)

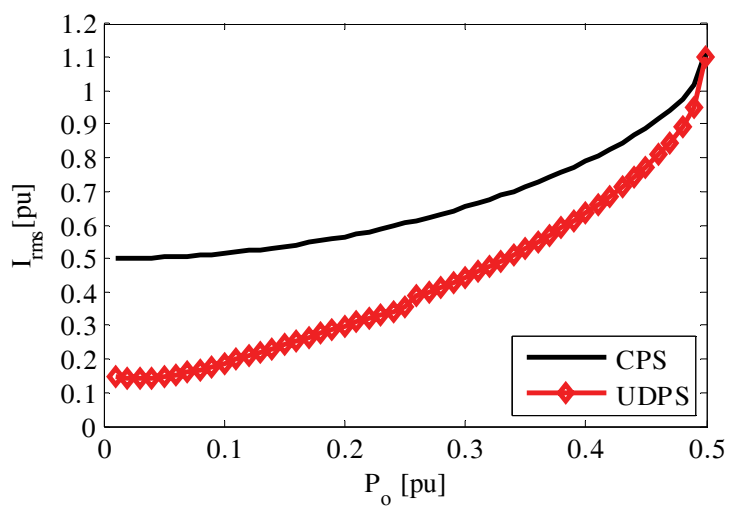

(b)

Fig. 9. Performance comparison with the UDPS scheme and CPS under the condition of $d=0.5$ in terms of the rms current, and the $P_{R L} / P_{o}$; (a) $P_{R L} / P_{o}$ versus $P_{o}$; (b) the inductor rms current

constraints for various modulation strategies are defined in order to determine whether the DAB converter operates in the soft-switching region [12]. If the constraints are fulfilled, only the switching-off losses are considered. Here, the complete calculation expression for $P_{s w}$ is shown as

$$
P_{-s w}=2\left(V_{S 1} I_{s w, Q_{11}, Q_{12}, Q_{13}, Q_{14}}+V_{S 2} I_{s w, Q_{21}, Q_{22}, Q_{23}, Q_{24}}\right)\left(t_{\text {on }}+t_{\text {off }}\right) f
$$

where $I_{s w}$ is the transition current of the MOSFETs, $t_{\text {on }}$ and $t_{\text {off }}$ represent the time intervals during switching on and off transients.

The loss of transformer and inductor $P_{m a g}$ includes the winding resistive losses $P_{c u}$ and the magnetic core losses $P_{\text {core }}$. Their expressions are given by:

$$
\begin{gathered}
P_{c u}=R_{L s} I_{L}^{2} \\
P_{\text {core }}=V_{c} k f^{\alpha} \widehat{B}^{\beta}
\end{gathered}
$$

where $R_{L s}$ is the equivalent winding resistance, $V_{c}$ is the core volume, $\hat{B}$ is the flux density, and $\mathrm{k}, \alpha$ and $\beta$ can be obtained in the core datasheet [21].

\section{Experimental Results}

A prototype of DAB converter was built up and the main parameters include: $V_{S l}=20 \mathrm{~V}, d=0.5 \sim 2, L_{s}=1.73 \mu \mathrm{H}, f_{s}=$ $100 \mathrm{kHz}$, and deadtime $T_{d}=0.2 \mu \mathrm{s}$. The primary devices are PSMN5R5-60YS and the secondary devices are STB13NM60N. The transformer turns ratio is 6 and the magnetic material is RM14-3F3 with leakage inductance $1.73 \mu \mathrm{H}$. The theoretical maximum output power with the proposed UDPS is expressed by [2]

$$
P_{\max }=\left(d V_{S 1}^{2}\right) /\left(8 L_{s} f_{s}\right)
$$

With the main parameters of the built prototype, the expression for the theoretical maximum output power with

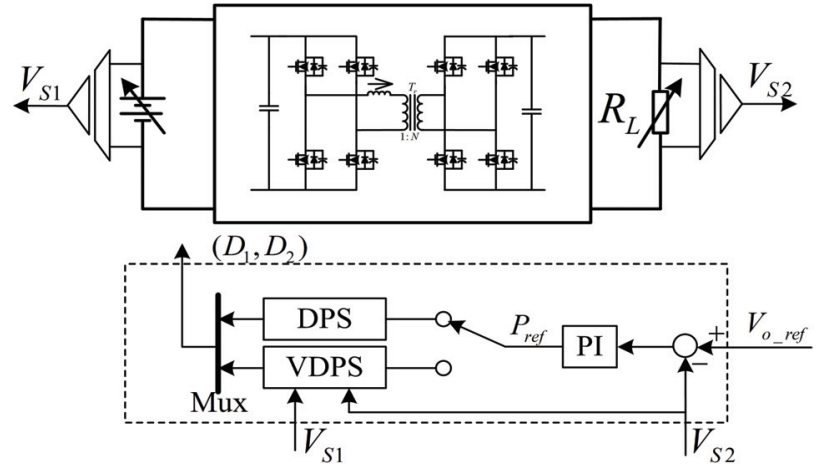

Fig. 10. Configuration of the experimental setup

the UDPS control is:

$$
P_{\max }=289 d
$$

It shows that the ideal maximum output power is the function of the voltage conversion ratio $d$. Furthermore, the non-ideal parameters such as the device voltage drop, the parasitic resistance, and the deadtime show effects on the actual output power range. The output power boundaries for various operating modes are also affected, which have been discussed in [22].

Fig. 10 shows the configuration of the experimental setup. A variable DC voltage source is used as input source and a variable resistive load is used as the load. The output voltage $V_{S 2}$ is regulated and the output reference is symbolled as $V_{\text {or ref }}$. The UDPS control scheme is adopted and each mode is selected by $P_{\text {ref }}$. The phase-shift pair $\left(D_{l}\right.$, $D_{2}$ ) is generated according to expression (24) and (25). DSP TMS320F2808 is used as the digital controller. A wide output power range is experimental measured with the built prototype.

\subsection{UDPS for low-power range}

Fig. 11(a) and (b) show the experimental waveform of 


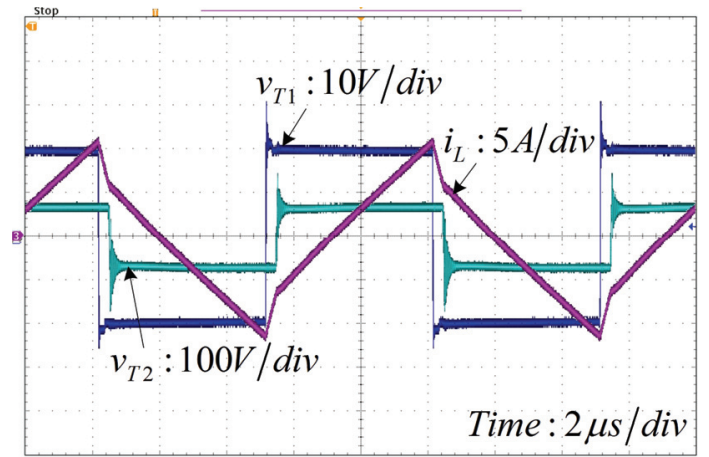

(a)

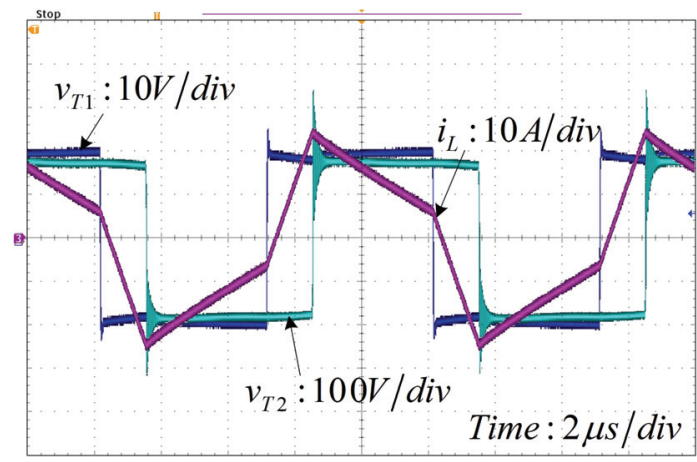

(c)

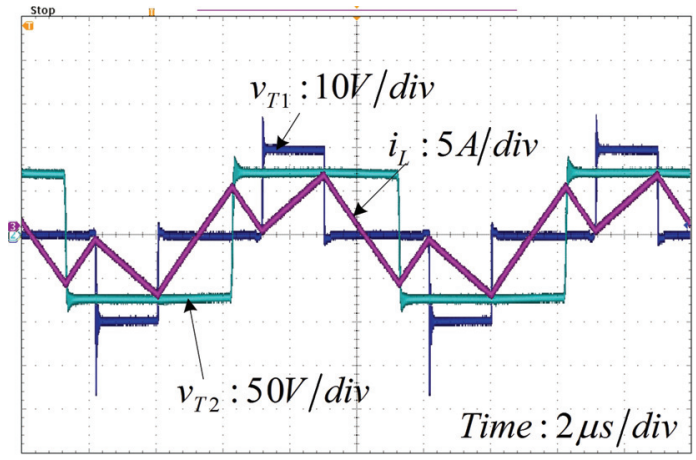

(b)

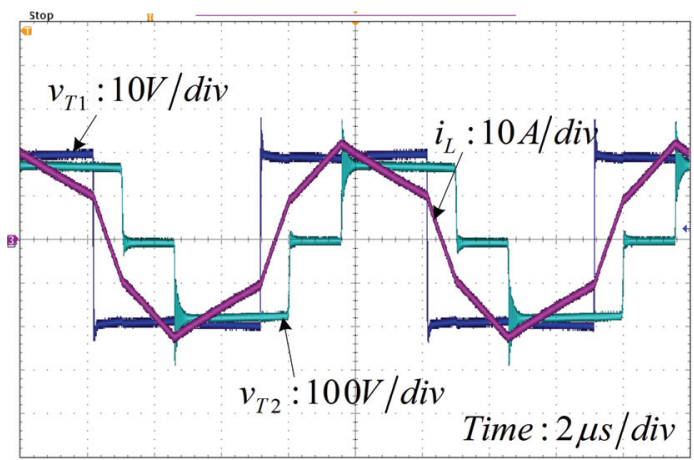

(d)

Fig. 11. Experimental results: (a) CPS under " $P_{\text {out }}=25 \mathrm{~W}, d=0.6$ "; (b) DPS_III under " $P_{\text {out }}=25 \mathrm{~W}, d=0.6$ "; (c) CPS under " $P_{\text {out }}=250 \mathrm{~W}, d=1.5$ "; (b) VDPS_I under " $P_{\text {out }}=250 \mathrm{~W}, d=1.5$ "

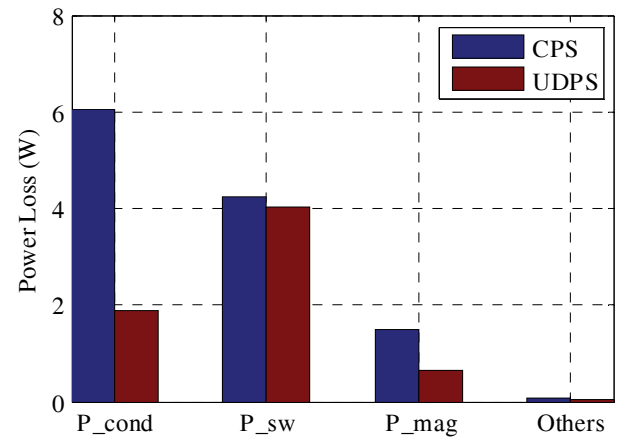

(a)

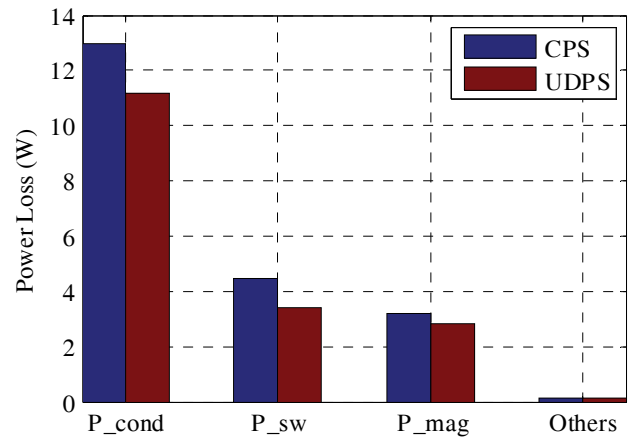

(b)

Fig. 12. Power loss distribution comparison with CPS and UDPS; (a) Low-power range: $P_{o}=25 \mathrm{~W}$; (b) High-power range: $P_{o}=250 \mathrm{~W}$

$v_{T 1}, v_{T 2}$, and $i_{L}$ with CPS and DPS_II respectively for buck operation. The voltage ratio $d$ is set as 0.6. According to (31), the ideal maximum output power is calculated as $173.4 \mathrm{~W}$. Here, the actual power of $25 \mathrm{~W}$ is used as the typical case for the low-power range.

In order to the performance comparison, the peak and rms values of $i_{L}$ are recorded. The measured inductor peakpeak and the rms values are $14 \mathrm{~A}$ and $3.55 \mathrm{~A}$ respectively with DPS_II control. With CPS, the measured peak-peak and the rms values of $i_{L}$ are $22.6 \mathrm{~A}$ and $5.89 \mathrm{~A}$. The measured efficiency with DPS_II is improved from $67.5 \%$ of CPS to $90.1 \%$. The efficiency improvement is more than $20 \%$ under this condition. It verifies the advantages of the proposed UDPS in minimization the inductor rms current and improving efficiency.

\subsection{UDPS for high-power range}

Fig. 11(c) and (d) illustrate the measured waveform of $v_{T 1}, v_{T 2}$, and $i_{L}$ using the CPS and VDPS I under " $d=1.5$ ". According to (31), the ideal maximum output power is calculated as $433.5 \mathrm{~W}$. Considering the effects of the non- 
ideal parameters such as the device voltage drop, the parasitic resistance, and the deadtime, here, the actual power of $250 \mathrm{~W}$ is used as the typical case for the highpower range. The measured efficiency is $91.9 \%$ of CPS in comparison to $92.8 \%$ of VDPS_I, respectively.

\subsection{Power loss distribution analysis}

The power loss breakdown of DAB converters with CPS and UDPS are calculated and compared in Fig. 12. Fig. 12 shows that $P_{\text {cond }}$ is the dominant component in the total power loss. Considering the advantage of UDPS in minimizing the reactive current, both $P_{c o n d}$ and $P_{\text {mag }}$ are significantly reduced.

\section{Conclusion}

This paper has discussed the reactive power losses of DAB converter by using various modulation strategies. Complete reactive components are included in the proposed model, including that delivered from the load and back to the source. With the defined reactive power loss as the optimization objective, the optimal operating mode is determined. In order to achieve a complete output power range, an unified dual-phase-shift (UDPS) scheme is proposed. The proposed UDPS has the same output power capability as the conventional phase-shift (CPS) method. Furthermore, the UDPS scheme is easy-to-implement since only the direction of the leading phase-shift D1 is changed for different operating power range. The reactive current distribution and inductor rms current with UDPS and CPS are compared through simulation and experiments results. In the experiments, a scale-down prototype was set up to show the advantages of the proposed method. The power loss distribution was illustrated and the efficiency improvement was verified with experiments.

\section{Acknowledgements}

This research was supported by University Research Development Fund (RDF-14-02-03), State Key Laboratory of Electrical Insulation and Power Equipment (EIPE15203), the Jiangsu Science and Technology Programme (BK20161252), and the National Nature Science Foundation of China (51407145).

\section{References}

[1] Engel, S.P.; Stieneker, M.; Soltau, N.; Rabiee, S.; Stagge, H.; De Doncker, R.W., "Comparison of the Modular Multilevel DC Converter and the DualActive Bridge Converter for Power Conversion in HVDC and MVDC Grids," IEEE Trans. Power
Electron., vol. 30, no. 1, pp. 124-137, Jan. 2015.

[2] Huiqing Wen; Weidong Xiao; Bin Su, "Nonactive Power Loss Minimization in a Bidirectional Isolated DC-DC Converter for Distributed Power Systems," IEEE Trans. Ind. Electron., vol. 61, no. 12, pp. 68226831, Dec. 2014.

[3] Ge, J.; Zhao, Z.; Yuan, L.; Lu, T., "Energy FeedForward and Direct Feed-Forward Control for Solid State Transformer," IEEE Trans. Power Electron., 2014, doi:10.1109/TPEL.2014.2382613.

[4] Hyun-Jun Choi, and Jee-Hoon Jung, "Practical Design of Dual Active Bridge Converter as Isolated Bidirectional Power Interface for Solid State Transformer Applications," Journal of Electrical Engineering \& Technology, vol. 11. No, 5, pp. 1265-1273, 2016.

[5] Krismer, F.; Kolar, J. W., "Efficiency-Optimized High-Current Dual Active Bridge Converter for Automotive Applications," IEEE Trans. Ind. Electron., vol. 59, no. 7, pp. 2745-2760, July 2012.

[6] Naayagi, R. T.; Forsyth, A. J.; Shuttleworth, R., "Bidirectional control of a dual active bridge DC-DC converter for aerospace applications," IET Power Electron., vol. 5, no. 7, pp. 1104-1118, August 2012.

[7] Rodriguez, A.; Vazquez, A.; Lamar, D. G.; Hernando, M. M.; Sebastian, J., "Different Purpose Design Strategies and Techniques to Improve the Performance of a Dual Active Bridge With Phase-Shift Control," IEEE Trans. Power Electron., vol. 30, no. 2, pp. 790-804, Feb. 2015.

[8] C. Mi, H. Bai, C. Wang, and S. Gargies, "Operation, design and control of dual H-bridge-based isolated bidirectional DC-DC converter," IET Power Electron., vol. 1, pp. 507-517, 2008.

[9] Engel, S. P.; Soltau, N.; Stagge, H.; De Doncker, R.W., "Improved Instantaneous Current Control for High-Power Three-Phase Dual-Active Bridge DCDC Converters," IEEE Trans. Power Electron., vol. 29, no. 8, pp. 4067-4077, Aug. 2014.

[10] Biao Zhao; Qiang Song; Wenhua Liu; Yandong Sun, "Overview of Dual-Active-Bridge Isolated Bidirectional DC-DC Converter for High-Frequency-Link Power-Conversion System," IEEE Trans. Power Electron., vol. 29, no. 8, pp. 4091-4106, Aug. 2014.

[11] Xiaodong Li; Yi-Fan Li, "An Optimized Phase-Shift Modulation For Fast Transient Response in a DualActive-Bridge Converter," IEEE Trans. Power Electron., vol. 29, no. 6, pp. 2661-2665, June 2014.

[12] G. G. Oggier, Garci, x, G. O. a, and A. R. Oliva, "Modulation Strategy to Operate the Dual Active Bridge DC-DC Converter Under Soft Switching in the Whole Operating Range," IEEE Trans. Power Electron., vol. 26, pp. 1228-1236, 2011.

[13] B. Hua and C. Mi, "Eliminate Reactive Power and Increase System Efficiency of Isolated Bidirectional Dual-Active-Bridge DC-DC Converters Using Novel Dual-Phase-Shift Control," IEEE Trans. Power 
Electron., vol. 23, pp. 2905-2914, 2008.

[14] Wen, H.; Su, B.; Xiao, W., "Design and performance evaluation of a bidirectional isolated dc-dc converter with extended dual-phaseshift scheme," IET Power Electron., vol. 6, no. 5, pp. 914-924, May 2013.

[15] Yu Du; Lukic, S. M.; Jacobson, B. S.; Huang, A. Q., "Modulation Technique to Reverse Power Flow for the Isolated Series Resonant DC-DC Converter With Clamped Capacitor Voltage," IEEE Trans. Ind. Electron., vol.59, no.12, pp. 4617-4628, Dec. 2012.

[16] Jong-Woo Kim; Gun-Woo Moon, "A New LLC Series Resonant Converter with a Narrow Switching Frequency Variation and Reduced Conduction Losses," IEEE Trans. Power Electron., vol. 29, no. 8, pp. 4278-4287, Dec. 2012.

[17] Wei Chen; Ping Rong; Zhengyu Lu, "Snubberless Bidirectional DC-DC Converter With New CLLC Resonant Tank Featuring Minimized Switching Loss," IEEE Trans. Ind. Electron., vol. 57, no. 9, pp. 30753086, Sept. 2010.

[18] Z. Biao, Y. Qingguang, and S. Weixin, "ExtendedPhase-Shift Control of Isolated Bidirectional DC-DC Converter for Power Distribution in Microgrid," IEEE Trans. Power Electron., vol. 27, pp. 4667-4680, 2012.

[19] M. Kim, M. Rosekeit, S.-K. Sul, and R. W. A. A. De Doncker, "A dual-phase-shift control strategy for dual-active-bridge DC-DC converter in wide voltage range," in Proc. IEEE ICPE \& ECCE, 2011, pp. 364371.

[20] Huiqing Wen, "Reactive power loss optimization method for bi-directional isolated DC-DC converters," in Proc. IEEE IPEC \& ECCE, 2014, pp. 702-706, 1821.

[21] W. A. Roshen, "A practical, accurate and very general core loss model for nonsinusoidal waveforms," IEEE Trans. Power Electron., vol. 22, no. 1, pp. 30-40, Jan. 2007.

[22] Huiqing Wen, Bin Su, "Operating modes and practical power flow analysis of bidirectional isolated power interface for distributed power systems," Energy Conversion and Management, vol. 111, pp. 229-238, 2016.

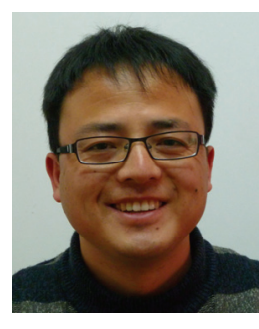

Huiqing Wen received the B.S. degree and M.S. degree in electrical engineering from Zhejiang University, Hangzhou, China, in 2002 and 2006 respectively. In 2009, he received the Ph.D. degree in electrical engineering at the Chinese Academy of Science, Beijing, China. From 2009 to 2010, he has been an Electrical Engineer working with the GE (China) Research and Development Center Company, Ltd., Shanghai, China. From 2010 to 2011, he was an engineer at the China Coal Research Institute, Beijing, China. From 2011 to 2012, he was a postdoctoral fellow at the Masdar Institute of Science and Technology, Abu Dhabi, United Arab Emirates. Currently he is an Associate Professor at the Xi'an Jiaotong-Liverpool University, Suzhou, China. His research interests include bidirectional DC-DC converter, power electronics in flexible ac transmission (FACTS) applications, electrical vehicles (EVs), and high power three-level electrical driving system.

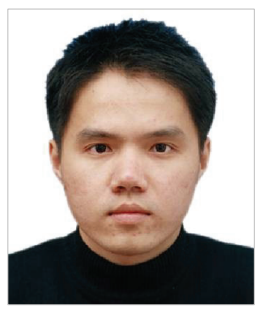

Bin Su was born in Wenzhou, China, in 1981. He received the Ph.D. degree in electrical engineering from Zhejiang University, Hangzhou, China, in 2010. He has authored or coauthored nine published technical papers. His research interests include topologies, modeling and control in power electronics. 